\title{
Performance Analysis of Feature Extraction Techniques for Facial Expression Recognition
}

\author{
Neha \\ Banasthali Vidyapeeth, \\ Banasthali, India
}

\author{
Pratistha Mathur \\ Banasthali Vidyapeeth, \\ Banasthali, India
}

\author{
Sandeep Kumar Gupta \\ Machine Learning Research \\ Lab, Jaipur, India
}

\begin{abstract}
Facial Expression Recognition is a vital topic for research in current scenario which has many applications as machine based HR interviews and human-machine interaction. Facial Expression recognition is applied for identification of person using face of a person. Researchers have proposed many research techniques for facial expression recognition but still accuracy, illumination and occlusion are the research issues which have to improve. So research issues are to improve recognition rate by improving the pre-processing of datasets, improving the feature extraction method and using the best classifier for facial expression recognition. Feature extraction is the key step on which recognition rate depends for facial expression recognition. The purpose of this research work is to analysis of different feature extraction technique in frequency domain as Gabor filter, Discrete Wavelet Transform and Discrete Cosine Transform feature extraction technique. Accuracy is the key research issue in facial expression recognition which is measured in term of Recognition rate.
\end{abstract}

\section{Keywords}

Facial expression recognition, Gabor Filter, DCT, DWT.

\section{INTRODUCTION}

The facial expression Recognition is considered as the important technique for biometric based gesture identification technologies which provides high uniqueness and stability with noninvasiveness, anti-falsification, robustness and some more features[1]. The methodology of facial expression recognition includes facial image acquisition, preprocessing, feature extraction and classification[2]. On the basis of domain, Facial expression recognition approach is classified into space domain based approach and frequency domain based approach [3]. Space domain based technique include local binary pattern, Principle component analysis and Gabor filter and frequency based approach includes discreet wavelet transform, discrete cosine transform and Fourier transform based feature extraction techniques [4]. DWT is used for image compression [5] and texture classification [6] due to its analytical capability of multiresolution decomposition. Fengjun Chen et al. [7] used 14 wavelet decomposed regions for enhance the accuracy of facial gesture recognition. Sameer S. Kulkarni et al. [8] analyzed impact of image block size using discrete cosine transform based feature extraction for facial expression recognition for determination of best block size to achieve best accuracy and explained different types of feature extraction algorithms based on discrete cosine transform (DCT).

In this paper we have analyzed DWT, gabor amplitude and DCT based feature extraction technique for facial expression recognition and implemented results are compare and discussed.

\section{RELATED WORK}

\subsection{Gabor Filter Feature Extraction} Technique

A Gabor filter is given as the following equation (1) [9].

$\Psi(\mathrm{x}, \mathrm{y}, \lambda, \theta)=\frac{1}{2 \Pi \mathrm{SxSy}} e^{-1 / 2\left(\frac{x^{\prime 2}}{S x * S x}+\frac{y^{\prime} 2}{S y * S y}\right)} e^{\mathrm{j} 2 \Pi \mathrm{x}^{\prime} / \lambda}$

In the equation (1), $(x, y)$, represents the pixel location in the digital image; $\lambda$ is wavelength, $\theta$ is projection orientation of Gabor kernel to image, $S_{\mathrm{x}}, S_{\mathrm{y}}$ Standard deviation in the $\mathrm{x} \& \mathrm{y}$ directions respectively. The parameters $\left(\mathrm{x}_{1}, \mathrm{y}_{1}\right)$ is gabor transformed positions which is generated using equation (2).

$\mathrm{x}_{1}=\mathrm{x} \cos \theta+\mathrm{y} \sin \theta \quad \mathrm{y}_{1}=-\mathrm{x} \cos \theta+\mathrm{y} \sin \theta$

The gabor kernel coefficients are generated using equation (1) and gabor kernel $\Psi(\mathrm{x}, \mathrm{y})$ is projected on input image $\mathrm{I}(\mathrm{x}, \mathrm{y})$ using convolution operation which is defined in equation (3) [10].

$\mathrm{G}_{\mathrm{u}, \mathrm{v}}(\mathrm{x}, \mathrm{y})=\mathrm{I}(\mathrm{x}, \mathrm{y}) * \Psi(\mathrm{x}, \mathrm{y})$

The Gabor kernel $\Psi(\mathrm{x}, \mathrm{y})$ is complex number so projection using convolution operation is evaluated separately for real and imaginary part as defined in equation 4 and 5 .

$\mathrm{R}(\mathrm{g}(\mathrm{x}, \mathrm{y}))_{\mathrm{i}, \mathrm{j}}=\mathrm{I}(\mathrm{x}, \mathrm{y}) *\left(\psi\left(\mathrm{x}, \mathrm{y}, \lambda_{\mathrm{m}}, \theta_{\mathrm{n}}\right)\right)_{\text {real }}$
$\mathrm{I}\left(\mathrm{g}(\mathrm{x}, \mathrm{y})_{\mathrm{i}, \mathrm{j}}=\mathrm{I}(\mathrm{x}, \mathrm{y}) *\left(\psi\left(\mathrm{x}, \mathrm{y}, \lambda_{\mathrm{m}}, \theta_{\mathrm{n}}\right)\right)_{\text {imaginary }}\right.$

Gabor filter is represented by equation (6).

$\mathrm{g}(\mathrm{x}, \mathrm{y})=\mathrm{R}(\mathrm{g}(\mathrm{x}, \mathrm{y}))+\mathrm{i} \mathrm{I}(\mathrm{g}(\mathrm{x}, \mathrm{y})$

The $\mathrm{g}(\mathrm{x}, \mathrm{y})$ is complex number and each coefficient of gabor amplitude is evaluated as equation 7 [11].

$\left.\lg (\mathrm{x}, \mathrm{y})\right|_{\mathrm{i}, \mathrm{j}}=\left(\left(\mathrm{R}(\mathrm{g}(\mathrm{x}, \mathrm{y}))_{\mathrm{i}, \mathrm{j}}\right)^{2}+\left(\mathrm{I}(\mathrm{g}(\mathrm{x}, \mathrm{y}))_{\mathrm{i}, \mathrm{j}}\right)^{2}\right)^{1 / 2}$

\subsection{Discrete Cosine Transform based Feature Extraction Technique}

DCT is a process to modify a signal into elementary frequency components. DCT is a closely related to discrete Fourier transform (DFT), using the DCT a signal is categorized into its basic frequency components. When we use DCT on $X^{*} Y$ sized matrix, the 2D-DCT extract the energy information of the image and then it will focus on some specific features located in the upper left Corner of the outcome real-valued $X^{*} Y$ DCT matrix Then the result matrix is used as a feature vector (FV) technique is used to improve the facial expression images. The recovery of images is practical just because of the DCT. The workings of DCT coefficients return the average energy of pixel blocks whereas the AC components return the intensity of image. As DCT separates an image hooked on discrete blocks of pixels of differing significance or weightage in an image so we can say that DCT is a lossy compression technique [12]. 
Discrete cosine transform is defined [13] as equation (8).

$$
\begin{aligned}
& F(i, j) \\
& =\alpha(i) \alpha(j) \sum_{x=0}^{A-1} \sum_{y=0}^{B-1} f(x, y) \cos \left[\frac{\pi(2 x+1) i}{2 A}\right] \cos \left[\frac{\pi(2 y+1) j}{2 B}\right] \\
& \text { whereu }=0,1,2, \ldots \ldots \ldots . . .1 \text { and } v= \\
& 0,1,2, \ldots \ldots \ldots \ldots N-1 \text {. }
\end{aligned}
$$

\subsection{Discrete Wavelet Transform based Feature Extraction Technique}

Recognition and image compression techniques wavelet transform gives an effective result. We can calculate Wavelet coefficients by a wavelet transform which shows changes according to time interval at a specific resolution. Taking the time interval makes it easy to calculate and remove the noise from image. The term wavelet transform is explained as decomposition of the data or the image into wavelet coefficients, comparing the detail coefficients with a given threshold value, and shrinking these coefficients close to zero to take away the effect of noise in the data. The image is reconstructed from the modified coefficients which are known as the inverse discrete wavelet transforms [14]. DWT transformation convert the facial expression image into four different frequency sub band as LL, LH, HL and HH as figure 1. Where range of frequency is represented as $\mathrm{LL}<\mathrm{LH}<\mathrm{HL}<\mathrm{HH}$. The feature or characteristics of facial expression is represented by low frequency coefficients or LL sub band so LL frequency sub band is extracted for further feature reduction [15].

\begin{tabular}{|c|c|}
\hline LL Sub band & LH Sub band \\
\hline HL Sub band & HH Sub band \\
\hline
\end{tabular}

Figure 1: frequency Sub band of DWT

\section{PROPOSED WORK}

This paper evaluated different feature extraction technique based on frequency domain on facial expression recognition. In the Gabor filter feature extraction, Gabor projection is applied on image but there is probability of increasing of redundancy which can be responsible of reducing the recognition rate. Gabor has a major problem issue of high dimension and high redundancy while it provides high energy difference of shape information and non shape information. These major issues should be reduced using some technique. The dimension reduction technique for gabor is called filtering so this whole technique is called gabor filter. The 7 different angles is applied for Gabor projection. The JAFFE dataset is used for experiment with ratio 70/30 of training/testing. The classification results of Gabor filter is compared with results of facial expression recognition method and compared with DWT and DCT based feature extraction technique.

In the proposed system, 2 different scales and 7 different orientations or total $5 * 2=14$, Gabor matrices are generated. The extraction features are reduced using principle component analysis (PCA) technique for facial expression recognition.

\section{EXPERIMENTS \& RESULTS}

The DCT, DWT and Gabor filter with PCA feature extraction Technique is evaluated in matlab with JAFFE dataset. Addaboost classifier is used for classification of 7 different expressions Natural, Surprise, Disgust, Happy, Fear, Sad, and Anger. Performance parameter is evaluated in term of recognition rate as shown equation 9 . The comparative results are shown in table 1 .

\begin{tabular}{|c|c|c|}
\hline S no. & Methods & $\begin{array}{c}\text { Avg. } \\
\text { Recognition } \\
\text { Rate }(\%)\end{array}$ \\
\hline 1. & Log Gabor + PCA[10] & 70 \\
\hline 2. & $\begin{array}{l}\text { Gabor filter + PCA based facial } \\
\text { expression recognition [11] }\end{array}$ & 80 \\
\hline 3. & $\begin{array}{l}\text { Principle Component Analysis } \\
{[16]}\end{array}$ & 77.78 \\
\hline 4 & $\begin{array}{l}\text { DWT based feature extraction } \\
\text { technique for facial expression } \\
\text { recognition }\end{array}$ & 81 \\
\hline 5. & $\begin{array}{l}\text { DCT feature extraction for } \\
\text { facial expression recognition }\end{array}$ & 80 \\
\hline 6. & $\begin{array}{l}\text { Gabor sampling } \text { Feature } \\
\text { selection for facial expression } \\
\text { recognition [17] }\end{array}$ & 67.5 \\
\hline 7. & $\begin{array}{l}\text { Gabor-DCT feature extraction } \\
\text { for facial expression } \\
\text { recognition [9] }\end{array}$ & 83.5 \\
\hline 7. & Gabor filter + PCA [11] & 80 \\
\hline 8. & $\begin{array}{c}\text { Proposed Scale Gabor filter + } \\
\text { PCA }\end{array}$ & 82.5 \\
\hline
\end{tabular}

Recognition Rate =correct expression matches/total tests (9)

Table 1. Comparative Accuracy of different feature extraction technique

\section{CONCLUSION}

From the results achieved by different technique, it is concluding that Gabor feature extractions have higher accuracy compared to discrete wavelet transform and discrete cosine transform. The analysis of better result of Gabor features, it is analyzed that Gabor is better extraction technique for edge or shape features extraction compared to DWT and DCT feature extraction. In DWT and DCT feature extraction, feature are extracted from Low frequency feature sub band (LL) and other frequency band are discarded so some of feature are lost which is cause of lower accuracy compare to Gabor. Gabor with scale projection achieved better accuracy compared to Gabor without scale. The problem with Gabor filter is generation of high redundant feature and huge dimension of feature matrix. 


\section{REFERENCES}

[1] Zheng Zhang "Wavelet Decomposition and Adaboost Feature Weighting For Facial Expression Recognition "International Conference on Control, Automation and Systems Engineering (CASE), pp.1-4,30-31,2011.

[2] Verma, Deepak, Vijaypal Dhaka, and Shubhlakshmi Agrwal. "An Improved average Gabor Wavelet filter Feature Extraction Technique for Facial Expression Recognition." International Journal on Innovations in Engineering and Technology 2 (2013): 2319-1058.

[3] Shilpa Choudhary, Kamlesh Lakhwani, and Shubhlakshmi Agrwal. "An efficient hybrid technique of feature extraction for facial expression recognition using AdaBoost Classifier." International Journal of Engineering Research \& Technology, issue: 1, vol no. 8 , 2012.

[4] Boles, Wageeh W., and Boualem Boashash. "A human identification technique using images of the iris and wavelet transform." IEEE transactions on signal processing 46, no. 4 (1998): 1185-1188.

[5] Soni, Karuna, Sandeep K. Gupta, Umesh Kumar, and Shubh L. Agrwal. "A new Gabor wavelet transform feature extraction technique for ear biometric recognition." In Power India International Conference (PIICON), 2014 6th IEEE, pp. 1-3. IEEE, 2014.

[6] Jyoti poonia, Parvati Bhurani, Rohit Kumar, Shubh Lakshmi Agrawal, "Performance Review of IRIS Recognition Systems", International Journal of Computer Systems (IJCS), 2(12), pp: 564-566, December 2015.

[7] Fengjun Chen, Zhiliang Wang, Zhengguang Xu, Donglin Wang, "Research on a Method of Facial Expression Recognition ", in IEEE The Ninth International Conference on Electronic Measurement \& Instruments, pp 225-230, IEEE, 2009.

[8] Kulkani, Sameer S., John Moriarty, and Chih-Cheng Hung. "The impact of Image block size on face feature extraction using discrete cosine transform." In IEEE Proceedings of the SoutheastCon 2010 (SoutheastCon), IEEE, 2010.

[9] Dosodia, Priya, Amarjeet Poonia, Sandeep K. Gupta, and Shubh Lakshmi Agrwal. "New Gabor-DCT feature extraction technique for facial expression recognition." In Communication Systems and Network Technologies (CSNT), 2015 Fifth International Conference on, pp. 546-549. IEEE, 2015.

[10] Lajevardi, Seyed Mehdi, and Margaret Lech. "Facial expression recognition using neural networks and loggabor filters." In Digital Image Computing: Techniques and Applications (DICTA), 2008, pp. 77-83. IEEE, 2008.

[11] Jun Ou, Xiao-Bo Bai*, Yun Pei ,Liang Ma, Wei Liu "Automatic Facial Expression Recognition Using Gabor Filter And Expression Analysis", in Second International Conference on Computer Modeling and Simulation, PP 216-218, IEEE, 2010.

[12] Tariq, Usman, and Thomas S. Huang. "Features and fusion for expression recognition-A comparative analysis." In 2012 IEEE Computer Society Conference on Computer Vision and Pattern Recognition Workshops, pp. 146-152. IEEE, 2012.

[13] Ahmed, Nasir, T. Natarajan, and Kamisetty R. Rao "Discrete cosines transform." IEEE transactions on Computers 100, no. 1 (1974): 90-93.

[14] Burrus, C. Sidney, Ramesh A. Gopinath, and Haitao Guo. "Introduction to wavelets and wavelet transforms: a primer." (1997).

[15] Jadhav, Dattatray V., and Raghunath S. Holambe "Feature extraction using Radon and wavelet transforms with application to face recognition."Neurocomputing 72, no. 7 (2009): 19511959 .

[16] Dongcheng, Shi, and Jiang Jieqing. "The method of facial expression recognition based on DWTPCA/LDA." In Image and Signal Processing (CISP), 2010 3rd International Congress on, vol. 4, pp. 19701974. IEEE, 2010

[17] Gupta, Sandeep K., ShubhLakshmi Agrwal, Yogesh K. Meena, and Neeta Nain. "A hybrid method of feature extraction for facial expression recognition." In SignalImage Technology and Internet-Based Systems (SITIS), 2011 Seventh International Conference on, pp. 422-425 IEEE, 2011. 